INDEPENDENT JOURNAL OF MANAGEMENT \& PRODUCTION (IJM\&P)

http://www.ijmp.jor.br

v. 11, n. 3, May-June 2020

ISSN: 2236-269X

DOI: 10.14807/ijmp.v11i3.1083

\title{
Multi objective fuzzy inventory model with deterioration, price and time dependent demand and time dependent holding cost
}

Satya Kumar Das

Govt. General Degree College at Gopiballavpur-II, India E-mail: satyakrdasmath75@gmail.com

Sahidul Islam

University of Kalyani, India

E-mail: sahidul.math@gmail.com

Submission: 6/25/2019

Revision: 9/18/2019

Accept: 10/2/2019

\section{ABSTRACT}

In this paper, we have formulated an inventory model with time dependent holding cost, selling price as well as time dependent demand. Multi-item inventory model has been considered under limitation on storage space. Due to uncertainty all the require cost parameters are taken as generalized trapezoidal fuzzy number. Our proposed multi-objective inventory model has been solved by using fuzzy programming techniques which are FNLP, FAGP, WFNLP and WFAGP methods. A numerical example is provided to demonstrate the application of the model. Finally to illustrate the model and sensitivity analysis and graphical representation have been shown.

Keywords: Inventory, Deterioration, Multi-item, Generalized trapezoidal fuzzy number, Fuzzy programming technique. 
ISSN: $2236-269 X$

DOI: 10.14807/ijmp.v11i3.1083

\section{INTRODUCTION}

Inventory model deals with decisions that minimize the total average cost or maximize the total average profit. In that way to construct a real life mathematical inventory model on base on various assumptions, notations and approximations.

In ordinary inventory system inventory costs i.e set-up cost, holding cost, deterioration cost, etc. are taken fixed amount but in real life inventory system these costs are not always fixed. So consideration of fuzzy variable is more realistic and interesting.

The inventory problems for deteriorating item such as fashionable items, electronics products, fruits, and green vegetables, and many others and the deterioration is defined as the spoilage, damage, dryness, vaporization etc. This results in decrease of usefulness of the commodity. Inventory problem for deteriorating items have been widely studied by many researchers. The economic order quantity model was first introduced in February 1913 by Harris.

Ghare and Schrader (1963) was the first to establish an economic order quantity (EOQ) model for deteriorating items. Then Covert and Philip (1973) extended their research work by presenting a variable rate of deterioration. Later, there are many papers presented on the deteriorating inventory, such as Sridevi et al. (2010), Bhunia and Shaikh (2014), and Ghosh, Sarkar and Chaudhuri (2015) etc. Kumar, et. al (2016) presented on Optimization of Weibull deteriorating items inventory model under the effect of price and time dependent demand with partial backlogging. Yang, H.L discussed on two warehouse partial backlogging inventory model for deteriorating items under inflation.

Yu-Chung Tsao, Gwo-Ji-Sheen (2008) studied on dynamic pricing promotion and replenishment policies for a deteriorating item under permissible delay in payments. Yang (2016) studied on two warehouse partial backlogging inventory model for deteriorating items under inflation. Liang and Zhou (2011) discussed on a two warehouse inventory model for deteriorating items under conditionally permissible delay in Payment.

The demand of an inventory item depends on the price is the most important in real life. Therefore the inventory system should incorporate the selling price as a decision variable. Bhunia and Shaikh (2014) presented a paper on deterministic inventory model for deteriorating items with selling price dependent demand and three-parameter Weibull distributed deterioration. Alfares and Ghaithan (2016) formulated on inventory and pricing model with price-dependent demand, time-varying holding cost, and quantity discounts. 
INDEPENDENT JOURNAL OF MANAGEMENT \& PRODUCTION (IJM\&P)

http://www.ijmp.jor.br

v. 11, n. 3, May-June 2020

ISSN: $2236-269 X$

DOI: 10.14807/ijmp.v11i3.1083

Shah et. al (2009) studied on a lot size inventory model for the Weibull distributed deterioration rate with discounted selling price and stock-dependent demand. Sridevi et. al (2010) discussed on Inventory model for deteriorating items with Weibull rate of replenishment and selling price dependent demand. The limitation of the space of the inventory item is the most important factor in the business management system.

Ghosh (2015) presented a paper on a multi-item inventory model for deteriorating items in limited storage space with stock-dependent demand. Islam and Mandal (2017), discussed fuzzy E.O.Q model with constant demand and shortages in a fuzzy signomial geometric programming (FSGP) approach. Mondal et. al (2003) formulated on an inventory system of ameliorating items for price dependent demand rate.

The concept of fuzzy set theory was first introduced by Zadeh in 1965. Afterward Zimmermann (1985) applied the fuzzy set theory concept with some useful membership functions to solve the linear programming problem with some objective functions. Then the various ordinary inventory model transformed to fuzzy versions model by various authors such as Roy and Maity (1995) presented on fuzzy inventory model with constraints.

Islam and Roy (2006) studied on a fuzzy EPQ model with flexibility and reliability consideration and demand depended unit production cost under a space constraint. Islam and Mandal (2017) discussed on fuzzy inventory model (EOQ model) with unit production cost, time depended holding cost, without shortages under a space constrain in a fuzzy parametric geometric programming (FPGP) approach. Maity (2008) developed a paper on fuzzy inventory model with two ware house under possibility measure in fuzzy goal. Roy (2014) presented on fuzzy inventory model for deteriorating items with price dependent demand.

In this paper, we have considered demand rate is depended on selling price as well as time and holding cost is time dependent. Multi-item inventory has been considered under limitation on storage space. Due to uncertainty all the required cost parameters are taken as generalized trapezoidal fuzzy number. The formulated multi objective inventory model has been solved by using FNLP, FAGP, WFNLP and WFAGP methods. A numerical example is considered to illustrate the model. Finally sensitivity analysis and graphical figures have been shown.

\section{MATHEMATICAL MODEL}

\subsection{Notations}

$c_{i}$ : Ordering cost per order for $\mathrm{i}^{\text {th }}$ item. 
DOI: 10.14807/ijmp.v11i3.1083

$H_{i}\left(=\alpha_{i} t\right)$ : Holding cost per unit and per unit time for $\mathrm{i}^{\text {th }}$ item.

$d_{i}$ : Deteriorating cost per unit and per unit time for $\mathrm{i}^{\text {th }}$ item.

$\theta_{i}$ : Constant deterioration rate per unit time for the $\mathrm{i}^{\text {th }}$ item.

$T_{i}$ : The length of cycle time for $\mathrm{i}^{\text {th }}$ item $\left(T_{i}>0\right)$.

$D\left(S_{i}, t\right)$ : Demand rate per unit time for the $\mathrm{i}^{\text {th }}$ item.

$S_{i}$ : Selling price for the $\mathrm{i}^{\text {th }}$ item.

$P_{i}$ : Purchasing cost for the $\mathrm{i}^{\text {th }}$ item.

$I_{i}(t)$ : Inventory level of the $\mathrm{i}^{\text {th }}$ items at time $t$.

$Q_{i}$ : The order quantity for the duration of a cycle of length $T_{i}$ for $\mathrm{i}^{\text {th }}$ item.

$\operatorname{TAP} P_{i}\left(T_{i}, S_{i}\right)$ : Total average profit per unit for the $\mathrm{i}^{\text {th }}$ item.

$w_{i}$ : Storage space per unit time for the $\mathrm{i}^{\text {th }}$ item.

$W$ : Total area of space.

$\widetilde{c_{l}}$ : Fuzzy ordering cost per order for the $\mathrm{i}^{\text {th }}$ item.

$\widetilde{\theta}_{l}$ : Fuzzy deterioration rate for the $\mathrm{i}^{\text {th }}$ item.

$\widetilde{P}_{l}$ : Purchasing cost for the $\mathrm{i}^{\text {th }}$ item in fuzziness.

$\widetilde{w_{l}}:$ Storage space per unit time for the $\mathrm{i}^{\text {th }}$ item in fuzziness.

$\widetilde{H_{l}}\left(=\widetilde{\alpha_{l}} t\right)$ : Fuzzy holding cost per unit per unit time for the $\mathrm{i}^{\text {th }}$ item

$\widetilde{d}_{l}$ : Fuzzy deteriorating cost per unit per unit time for the $\mathrm{i}^{\text {th }}$ item.

$\left.T A \widetilde{P_{l}\left(T_{l}\right.} S_{l}\right)$ : Fuzzy total average profit per unit for the $\mathrm{i}^{\text {th }}$ item.

$\widehat{c_{l}}$ : Defuzzyfication of fuzzy ordering cost per order for the $\mathrm{i}^{\text {th }}$ item.

$\widehat{\theta}_{l}$ : Defuzzyfication of fuzzy deterioration rate for the $\mathrm{i}^{\text {th }}$ item.

$\widehat{P}_{l}$ : Defuzzyfication of fuzzy purchasing cost for the $\mathrm{i}^{\text {th }}$ item.

$\widehat{W}_{l}$ : Defuzzyfication of fuzzy storage space per unit time for the $\mathrm{i}^{\text {th }}$ item.

$\widehat{H_{l}}\left(=\widehat{\alpha_{l}} t\right)$ : Defuzzyfication of fuzzy holding cost per unit per unit time for the $\mathrm{i}^{\text {th }}$ item

$\widehat{d}_{l}$ : Defuzzyfication of fuzzy deteriorating cost per unit per unit time for the $\mathrm{i}^{\text {th }}$ item. 
DOI: 10.14807/ijmp.v11i3.1083

$\left.T A \widehat{P_{l}\left(T_{l}\right.} S_{l}\right)$ : Defuzzyfication of fuzzy total average profit per unit for the $\mathrm{i}^{\text {th }}$ item.

\subsection{Assumptions}

1. The inventory system involves multi-item.

2. The replenishment occurs instantaneously at infinite rate.

3. The lead time is negligible.

4. Shortages are not allowed.

5. Demand rate is time depended as well as selling price. It is taken as $D\left(S_{i}, t\right)=\left(a_{i}-\right.$ $\left.b_{i} S_{i}\right) e^{\lambda_{i} t}$ where $a_{i}, b_{i}>0,\left(a_{i}-b_{i} S_{i}\right)>0$ and $S_{i} \geq P_{i}$.

6. Deterioration rate per unit time per cycle is $\theta_{i}$ for the $\mathrm{i}^{\text {th }}$ item. $0 \leq \theta_{i} \leq 1, \theta_{i}$ is constant.

\subsection{Model Formulation}

The inventory model for $\mathrm{i}^{\text {th }}$ item is illustrated in Figure-1. During the period $\left[0, T_{i}\right]$ the inventory level reduces due to demand rate and deterioration rate for $\mathrm{i}^{\text {th }}$ item. In this time period, the inventory level is described by the differential equation-

$\frac{d I_{i}(t)}{d t}+I_{i}(t) \theta_{i}=-D\left(S_{i}, t\right)=-\left(a_{i}-b_{i} S_{i}\right) e^{\lambda_{i} t}, \quad 0 \leq t \leq T_{i}$

With boundary condition, $I_{i}(0)=Q_{i}, I_{i}\left(T_{i}\right)=0$.

Solving (1) we have,

$$
\begin{aligned}
I_{i}(t) & =e^{-\theta_{i} t} \frac{\left(a_{i}-b_{i} S_{i}\right)}{\left(\theta_{i}+\lambda_{i}\right)}\left[e^{\left(\theta_{i}+\lambda_{i}\right) T_{i}}-e^{\left(\theta_{i}+\lambda_{i}\right) t}\right] \\
Q_{i} & =\frac{\left(a_{i}-b_{i} S_{i}\right)}{\left(\theta_{i}+\lambda_{i}\right)}\left(e^{\left(\theta_{i}+\lambda_{i}\right) T_{i}}-1\right)
\end{aligned}
$$

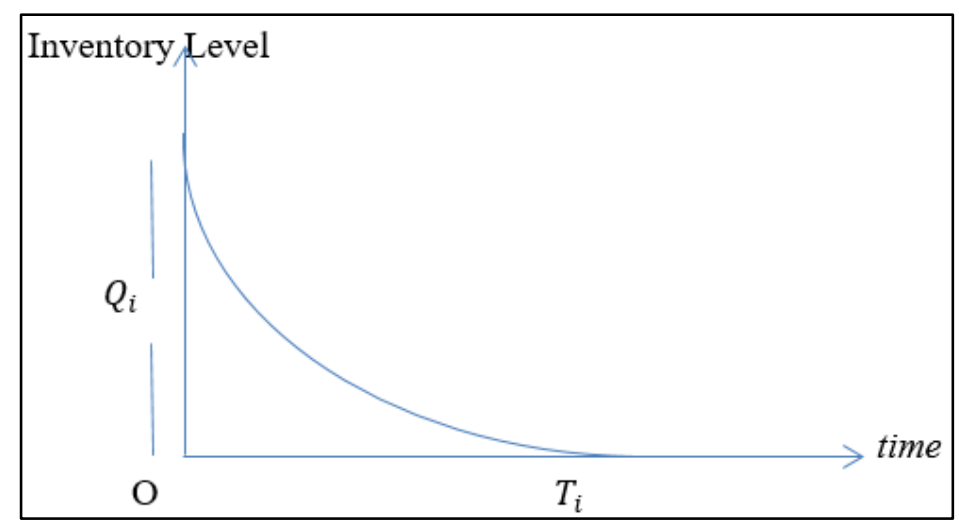

Figure 1: Inventory level for the $\mathrm{i}^{\text {th }}$ item. 
ISSN: 2236-269X

DOI: 10.14807/ijmp.v11i3.1083

Now we are calculating various costs for $i^{\text {th }}$ item as following

i) Sales revenue $\left(S R_{i}\right)=S_{i} \int_{0}^{T_{i}} D\left(S_{i}, t\right) d t$

$=\frac{S_{i}\left(a_{i}-b_{i} S_{i}\right)}{\lambda_{i}}\left(e^{\lambda_{i} T_{i}}-1\right)$

ii) Purchasing cost $\left(P C_{i}\right)=P_{i} Q_{i}=\frac{P_{i}\left(a_{i}-b_{i} S_{i}\right)}{\left(\theta_{i}+\lambda\right)}\left(e^{\left(\theta_{i}+\lambda\right) T_{i}}-1\right)$

iii) Inventory holding cost $\left(H C_{i}\right)=\int_{0}^{T_{i}} \alpha_{i} t I_{i}(t) d t$

$$
=\frac{\alpha_{i}\left(a_{i}-b_{i} S_{i}\right)}{\left(\theta_{i}+\lambda_{i}\right)}\left[-e^{\left(\theta_{i}+\lambda_{i}\right) T_{i}}\left\{\frac{T_{i} e^{-\theta_{i} T_{i}}}{\theta_{i}}+\frac{1}{\theta_{i}^{2}}\left(e^{-\theta_{i} T_{i}}-1\right)\right\}-\frac{T_{i} e^{\lambda T_{i}}}{\lambda_{i}}+\frac{1}{\lambda_{i}{ }^{2}}\left(e^{\lambda_{i} T_{i}}-1\right)\right]
$$

iv) Deterioration cost $\left(D C_{i}\right)=d_{i} \theta_{i} T_{i}$

v) Ordering cost $\left(O C_{i}\right)=c_{i}$

Total average profit per unit time for $i t h$ item

$$
\begin{aligned}
& \operatorname{TAP}_{i}\left(T_{i}, S_{i}\right)=\frac{1}{T_{i}}\left[S R_{i}-\left(P C_{i}+H C_{i}+D C_{i}+O C_{i}\right)\right] \\
& =\frac{S_{i}\left(a_{i}-b_{i} S_{i}\right)}{\lambda_{i} T_{i}}\left(e^{\lambda_{i} T_{i}}-1\right)-\frac{P_{i}\left(a_{i}-b_{i} S_{i}\right)}{\left(\theta_{i}+\lambda_{i}\right) T_{i}}\left(e^{\left(\theta_{i}+\lambda_{i}\right) T_{i}}-1\right)-\frac{\alpha_{i}\left(a_{i}-b_{i} S_{i}\right)}{\left(\theta_{i}+\lambda_{i}\right) T_{i}}\left[e ^ { ( \theta _ { i } + \lambda _ { i } ) T _ { i } } \left\{\frac{T_{i} e^{-\theta_{i} T_{i}}}{\theta_{i}}+\right.\right. \\
& \left.\left.\frac{1}{\theta_{i}^{2}}\left(e^{-\theta_{i} T_{i}}-1\right)\right\}-\frac{T_{i} e^{\lambda T_{i}}}{\lambda_{i}}+\frac{1}{\lambda_{i}{ }^{2}}\left(e^{\lambda_{i} T_{i}}-1\right)\right]-d_{i} \theta_{i}-\frac{c_{i}}{T_{i}}
\end{aligned}
$$

Multi-objective inventory model (MOIM) can be written as:

$\operatorname{Max}\left\{T A P_{1}\left(T_{1}, S_{1}\right), T A P_{2}\left(T_{2}, S_{2}\right), \ldots \ldots \ldots, T A P_{n}\left(T_{n}, S_{n}\right)\right\}$

Subject to, $\sum_{i=1}^{n} w_{i} Q_{i} \leq W, P_{i} \leq S_{i} \leq \frac{a_{i}}{b_{i}}$

where $\operatorname{TAP}_{i}\left(T_{i}, S_{i}\right)=\frac{S_{i}\left(a_{i}-b_{i} S_{i}\right)}{\lambda_{i} T_{i}}\left(e^{\lambda_{i} T_{i}}-1\right)-\frac{P_{i}\left(a_{i}-b_{i} S_{i}\right)}{\left(\theta_{i}+\lambda_{i}\right) T_{i}}\left(e^{\left(\theta_{i}+\lambda_{i}\right) T_{i}}-1\right)-$

$\frac{\alpha_{i}\left(a_{i}-b_{i} S_{i}\right)}{\left(\theta_{i}+\lambda_{i}\right) T_{i}}\left[e^{\left(\theta_{i}+\lambda_{i}\right) T_{i}}\left\{\frac{T_{i} e^{-\theta_{i} T_{i}}}{\theta_{i}}+\frac{1}{\theta_{i}^{2}}\left(e^{-\theta_{i} T_{i}}-1\right)\right\}-\frac{T_{i} e^{\lambda T_{i}}}{\lambda_{i}}+\frac{1}{\lambda_{i}{ }^{2}}\left(e^{\lambda_{i} T_{i}}-1\right)\right]-d_{i} \theta_{i}-\frac{c_{i}}{T_{i}}$

and $Q_{i}=\frac{\left(a_{i}-b_{i} S_{i}\right)}{\left(\theta_{i}+\lambda_{i}\right)}\left(e^{\left(\theta_{i}+\lambda_{i}\right) T_{i}}-1\right)$ for $i=1,2, \ldots \ldots \ldots n$

\subsection{Fuzzy Model}

Due to uncertainty, we assume all parameters as generalized trapezoidal fuzzy number (GTrFN). Let us assume, 
DOI: 10.14807/ijmp.v11i3.1083

$$
\begin{aligned}
& {\widetilde{a_{l}}}=\left(a_{i}{ }^{1}, a_{i}{ }^{2}, a_{i}{ }^{3}, a_{i}{ }^{4} ; w_{a_{i}}\right), 0<w_{a_{i}} \leq 1 ;{\widetilde{b_{l}}}=\left(b_{i}{ }^{1}, b_{i}{ }^{2}, b_{i}{ }^{3}, b_{i}{ }^{4} ; w_{b_{i}}\right), 0<w_{b_{i}} \leq 1 ; \\
& \widetilde{\theta}_{\iota}=\left(\theta_{i}{ }^{1}, \theta_{i}{ }^{2}, \theta_{i}{ }^{3}, \theta_{i}{ }^{4} ; w_{\theta_{i}}\right), 0<w_{\theta_{i}} \leq 1 ; \widetilde{\alpha_{l}}=\left(\alpha_{i}{ }^{1}, \alpha_{i}{ }^{2}, \alpha_{i}{ }^{3}, \alpha_{i}{ }^{4} ; w_{\alpha_{i}}\right), 0<w_{\alpha_{i}} \leq 1 ; \\
& \widetilde{d}_{\iota}=\left(d_{i}{ }^{1}, d_{i}{ }^{2}, d_{i}{ }^{3}, d_{i}{ }^{4} ; w_{d_{i}}\right), 0<w_{d_{i}} \leq 1 ; \widetilde{P}_{l}=\left(P_{i}{ }^{1}, P_{i}{ }^{2}, P_{i}{ }^{3}, P_{i}{ }^{4} ; w_{P_{i}}\right), 0<w_{P_{i}} \leq 1 ; \\
& {\widetilde{w_{l}}}=\left(w_{i}{ }^{1}, w_{i}{ }^{2}, w_{i}{ }^{3}, w_{i}{ }^{4} ; w_{w_{i}}\right), 0<w_{w_{i}} \leq 1 ; \widetilde{c_{l}}=\left(c_{i}{ }^{1}, c_{i}{ }^{2}, c_{i}{ }^{3}, c_{i}{ }^{4} ; w_{c_{i}}\right), 0<w_{c_{i}} \leq 1
\end{aligned}
$$

Then due to uncertainty, the total average profit for the $\mathrm{i}^{\text {th }}$ item is given by,

$\left.T A \widetilde{P_{l}\left(T_{l}\right.}, S_{l}\right)=\frac{\widetilde{P}_{l}\left(\tilde{a}_{i}-\widetilde{b}_{l} S_{i}\right)}{\left(\widetilde{\theta}_{l}+\lambda_{i}\right) T_{i}}\left(1-e^{\left(\widetilde{\theta}_{l}+\lambda_{i}\right) T_{i}}\right)+\frac{S_{i}\left(\tilde{a}_{i}-\widetilde{b}_{l} S_{i}\right)}{\lambda_{i} T_{i}}\left(e^{\lambda_{i} T_{i}}-1\right)-$

$\frac{\widetilde{\alpha_{l}}\left(\tilde{a}_{i}-\widetilde{b}_{l} S_{i}\right)}{\left(\widetilde{\theta_{l}}+\lambda_{i}\right) T_{i}}\left[e^{\left(\widetilde{\theta_{l}}+\lambda_{i}\right) T_{i}}\left\{\frac{T_{i} e^{-\widetilde{\theta_{l}} T_{i}}}{\widetilde{\theta_{l}}}+\frac{1}{{\widetilde{\theta_{l}}}^{2}}\left(e^{-\widetilde{\theta}_{l} T_{i}}-1\right)\right\}-\frac{T_{i} e^{\lambda_{i} T_{i}}}{\lambda_{i}}+\frac{1}{\lambda_{i}{ }^{2}}\left(e^{\lambda_{i} T_{i}}-1\right)\right]-\widetilde{d_{l}} \widetilde{\theta}_{l}-\frac{\widetilde{c_{l}}}{T_{i}}(7)$

and $Q_{i}=\frac{\left(\tilde{a}_{i}-\widetilde{b}_{l} S_{i}\right)}{\left(\widetilde{\theta}_{l}+\lambda_{i}\right)}\left(e^{\left(\widetilde{\theta_{l}}+\lambda_{i}\right) T_{i}}-1\right)$ for $i=1,2, \ldots \ldots \ldots, n$.

And our MOIM problem becomes fuzzy model as

$\left.\left.\left.\left.\operatorname{Max}\left\{T A \widetilde{P_{1}\left(T_{1}\right.}, S_{1}\right), T A \widetilde{P_{2}\left(T_{2}\right.}, S_{2}\right), T A \widetilde{P_{3}\left(T_{3}\right.}, S_{3}\right), \ldots \ldots \ldots \ldots, T A \widetilde{P_{n}\left(T_{n}\right.}, S_{n}\right)\right\}$

Subject to, $\sum_{i=1}^{n} \widetilde{w_{l}} Q_{i} \leq W, \widetilde{P_{l}} \leq S_{i} \leq \frac{\widetilde{a_{l}}}{\widetilde{b_{l}}}$

where $\left.T A \widetilde{P_{l}\left(T_{l}\right.}, S_{l}\right)=\frac{\widetilde{P_{l}\left(\tilde{a}_{i}-\widetilde{b}_{l} S_{i}\right)}}{\left(\widetilde{\theta}_{l}+\lambda_{i}\right) T_{i}}\left(1-e^{\left(\widetilde{\theta_{l}}+\lambda_{i}\right) T_{i}}\right)+\frac{S_{i}\left(\tilde{a}_{i}-\widetilde{b}_{l} S_{i}\right)}{\lambda_{i} T_{i}}\left(e^{\lambda_{i} T_{i}}-1\right)-$

$\frac{\widetilde{\alpha_{l}}\left(\tilde{a}_{i}-\widetilde{b}_{l} S_{i}\right)}{\left(\widetilde{\theta}_{l}+\lambda_{i}\right) T_{i}}\left[e^{\left(\widetilde{\theta_{l}}+\lambda_{i}\right) T_{i}}\left\{\frac{T_{i} e^{-\widetilde{\theta_{l}} T_{i}}}{\widetilde{\theta_{l}}}+\frac{1}{{\widetilde{\theta_{l}}}^{2}}\left(e^{-\widetilde{\theta}_{l} T_{i}}-1\right)\right\}-\frac{T_{i} e^{\lambda_{i} T_{i}}}{\lambda_{i}}+\frac{1}{\lambda_{i}{ }^{2}}\left(e^{\lambda_{i} T_{i}}-1\right)\right]-{\widetilde{d_{l}}}_{\imath} \widetilde{\theta}_{l}-\frac{\widetilde{c_{l}}}{T_{i}}$

and $Q_{i}=\frac{\left(\tilde{a}_{i}-\widetilde{b}_{l} S_{i}\right)}{\left(\widetilde{\theta}_{l}+\lambda_{i}\right)}\left(e^{\left(\widetilde{\theta_{l}}+\lambda_{i}\right) T_{i}}-1\right)$ for $i=1,2, \ldots \ldots \ldots, n$.

\section{MATHEMATICAL ANALYSIS}

\subsection{Ranking fuzzy numbers with respect to their total integral value.}

Ranking fuzzy number is an important aspect of decision making in a fuzzy environment. In reality, decision-makers having different viewpoints will give different ranking outcomes under the same situation. Bortolan and Degani (1985) studied the number of methods for ranking fuzzy numbers. Liou and Wang (1992) proposed a relatively simple computation and easily understood method.

Let $\lambda \in[0,1]$ be a pre-assigned parameter which is called the degree of optimism. The graded mean value (or, total $\lambda$-integer value) of generalized trapezoidal fuzzy number (GTrFN) is discussed below. 
DOI: 10.14807/ijmp.v11i3.1083

A fuzzy number $\tilde{A}=(a, b, c, d ; w)$ is called generalized trapezoidal fuzzy number (GTrFN) if its membership function is given by

$$
\mu_{\tilde{A}}^{w}(x)=\left\{\begin{array}{cc}
0 & \text { if } x \leq a \\
w \frac{x-a}{b-a} & \text { if } a \leq x \leq b \\
w & \text { if } b \leq x \leq c \\
w \frac{d-x}{d-c} & \text { if } c \leq x \leq d \\
0 & \text { if } d \leq x
\end{array}\right.
$$

The total $\lambda$ integer value of $\tilde{A}=(a, b, c, d ; w)$ is given as

$I_{\lambda}^{w}(\tilde{A})=\lambda I_{R}^{w}(\tilde{A})+(1-\lambda) I_{L}^{w}(\tilde{A})$, where $I_{L}^{w}(\tilde{A}), I_{R}^{w}(\tilde{A})$ are the left and right interval values of $\widetilde{A}$ respectively. Where $I_{L}^{w}(\tilde{A})=\int_{0}^{1}\left(\mu_{L \tilde{A}}^{w}\right)^{-1} \alpha d \alpha, \quad I_{R}^{w}(\tilde{A})=\int_{0}^{1}\left(\mu_{R \tilde{A}}^{w}\right)^{-1} \alpha d \alpha$

Now, $\left(\mu_{L \tilde{A}}^{w}\right)^{-1} \alpha=a+\frac{\alpha}{w}(b-a) \&\left(\mu_{R \tilde{A}}^{w}\right)^{-1} \alpha=d-\frac{\alpha}{w}(d-c)$

So the left \& right interval values $\widetilde{A}$ are

$I_{L}^{w}(\tilde{A})=w\left(\frac{a+b}{2}\right)$ and $I_{R}^{w}(\tilde{A})=w\left(\frac{c+d}{2}\right)$

Hence the total $\lambda$-integral value of $\tilde{A}$ is

$I_{\lambda}^{w}(\tilde{A})=\lambda w\left(\frac{c+d}{2}\right)+(1-\lambda) w\left(\frac{a+b}{2}\right)$

When $\lambda=1$, we obtain $I_{1}^{w}(\tilde{A})=I_{R}^{w}(\tilde{A})=w\left(\frac{c+d}{2}\right)$ which reflects an optimistic viewpoint.

When $\lambda=0$, we get $I_{0}^{w}(\tilde{A})=I_{L}^{w}(\tilde{A})=w\left(\frac{a+b}{2}\right)$ which reflects a pessimistic point of view. When $\lambda=0.5$ the total $\lambda$-integral value is

$I_{0.5}^{w}(\tilde{A})=\frac{1}{2}\left[I_{L}^{w}(\tilde{A})+I_{R}^{w}(\tilde{A})\right]=\frac{1}{2}\left[w\left(\frac{a+b}{2}\right)+w\left(\frac{c+d}{2}\right)\right]=w\left(\frac{a+b+c+d}{4}\right)$.

Which reflects a reasonably optimistic decision-makers perspective and is treated as the defuzzification of $\tilde{A}$.

\subsection{Fuzzy Model Using Defuzzification of Fuzzy Number.}

Using defuzzification of fuzzy number technique (9), we have the approximated values $\left(\widehat{c}_{l}, \widehat{a}_{l}, \widehat{b}_{l}, \widehat{\theta}_{l}, \widehat{\alpha}_{l}, \widehat{d}_{l}, \widehat{P}_{l}, \widehat{w}_{l}\right)$ of the GTrFN parameters $\left(\widetilde{c}_{l}, \widetilde{a}_{l}, \widetilde{b}_{l}, \widetilde{\theta}_{l}, \widetilde{\alpha}_{l}, \widetilde{d}_{l}, \widetilde{P}_{l}, \widetilde{w}_{l}\right)$.

So the above problem (8) reduces to

$\left.\left.\left.\left.\operatorname{Max}\left\{T A \widehat{P_{1}\left(T_{1}\right.}, S_{1}\right), T A \widehat{P_{2}\left(T_{2}\right.}, S_{2}\right), T A \widehat{P_{3}\left(T_{3}\right.}, S_{3}\right), \ldots \ldots \ldots \ldots \ldots \ldots, T A \widehat{P_{n}\left(T_{n}\right.}, S_{n}\right)\right\}$ 
ISSN: 2236-269X

DOI: 10.14807/ijmp.v11i3.1083

Subject to, $\sum_{i=1}^{n} \widehat{w_{l}} Q_{i} \leq W, \widehat{P_{l}} \leq S_{i} \leq \frac{\widehat{a_{l}}}{\widehat{b}_{l}}$

where $\left.T A \widehat{P_{l}\left(T_{l}\right.}, S_{l}\right)=\frac{\widehat{P}_{l}\left(\hat{a}_{i}-\widehat{b}_{l} S_{i}\right)}{\left(\widehat{\theta}_{l}+\lambda_{i}\right) T_{i}}\left(1-e^{\left(\widehat{\theta}_{l}+\lambda_{i}\right) T_{i}}\right)+\frac{S_{i}\left(\hat{a}_{i}-\widehat{l}_{l} S_{i}\right)}{\lambda_{i} T_{i}}\left(e^{\lambda_{i} T_{i}}-1\right)-$

$\frac{\widehat{\alpha_{l}}\left(\hat{a}_{i}-\widehat{b}_{l} S_{i}\right)}{\left(\widehat{\theta}_{l}+\lambda_{i}\right) T_{i}}\left[e^{\left(\widehat{\theta}_{l}+\lambda_{i}\right) T_{i}}\left\{\frac{T_{i} e^{-\widehat{\theta}_{l} T_{i}}}{\widehat{\theta}_{l}}+\frac{1}{{\widehat{\theta_{l}}}^{2}}\left(e^{-\widehat{\theta}_{l} T_{i}}-1\right)\right\}-\frac{T_{i} e^{\lambda_{i} T_{i}}}{\lambda_{i}}+\frac{1}{\lambda_{i}{ }^{2}}\left(e^{\lambda_{i} T_{i}}-1\right)\right]-\widehat{d_{l}} \widehat{\theta_{l}}-\frac{\widehat{c_{l}}}{T_{i}}$

and $Q_{i}=\frac{\left(\hat{a}_{i}-\widehat{b}_{l} S_{i}\right)}{\left(\widetilde{\theta}_{l}+\lambda_{i}\right)}\left(e^{\left(\widehat{\theta}_{l}+\lambda_{i}\right) T_{i}}-1\right)$ for $i=1,2, \ldots \ldots \ldots, n$.

\section{FUZZY PROGRAMMING TECHNIQUE (BASED ON MAX-MIN AND MAX- ADDITIVE OPERATORS) TO SOLVE MOIM}

Solve the MOIM (10) as a single objective using only one objective at a time and we ignoring the others. So we get the ideal solutions.

From the above results, we find out the corresponding values of every objective function at each solution obtained. With these values the pay-off matrix can be prepared as follows:

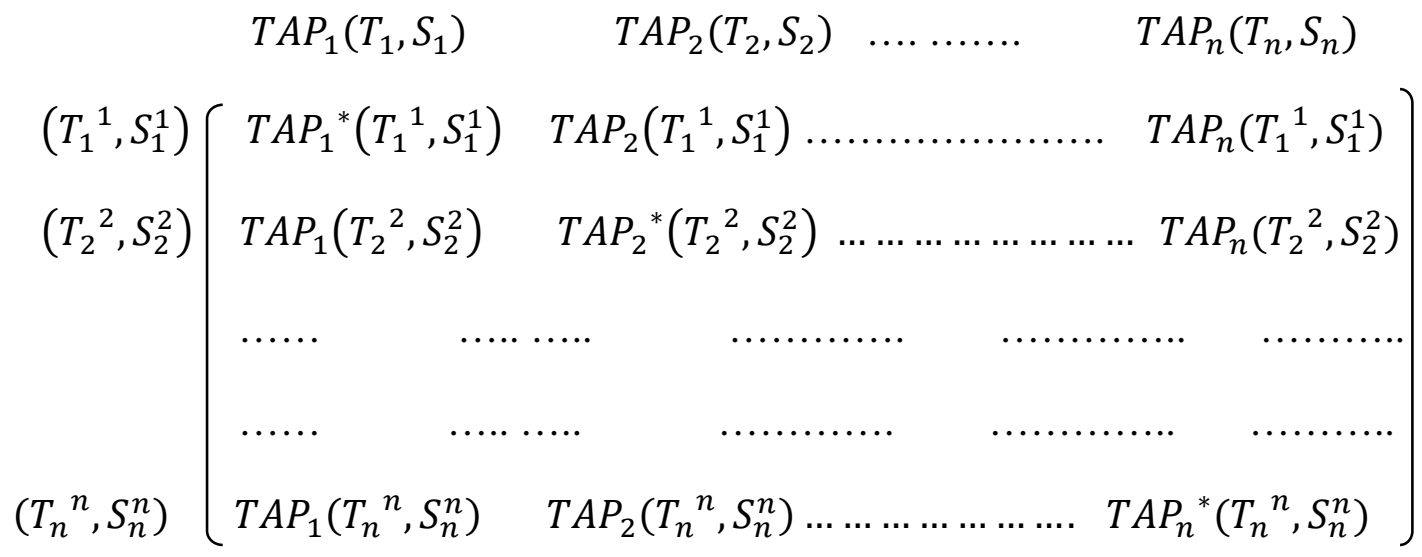

Let $L^{k}=\min \left\{\operatorname{TAP}_{k}\left(T_{i}{ }^{i}, S_{i}^{i}\right), i=1,2, \ldots, n\right\}$ for $k=1,2, \ldots, n$ and

$U^{k}=\operatorname{TAP}_{k}^{*}\left(T_{k}{ }^{k}, S_{k}^{k}\right)$ for $k=1,2, \ldots, n$.

Hence $\quad U^{k}, L^{k} \quad$ are identified, $L^{k} \leq T A P_{k}\left(T_{i}^{i}, S_{i}^{i}\right) \leq U^{k}$, for $i=1,2, \ldots, n ; k=$ $1,2, \ldots, n$.

Therefore fuzzy linear membership functions $\mu_{T A P_{k}}\left(\operatorname{TAP}_{k}\left(T_{k}, S_{k}\right)\right)$ for the $\mathrm{k}^{\text {th }}$ objective functions $\operatorname{TAP}_{k}\left(T_{k}, S_{k}\right)$ respectively for $k=1,2, \ldots, n$ are defined as follows:

$\mu_{T_{A P_{k}}}\left(\operatorname{TAP}_{k}\left(T_{k}, S_{k}\right)\right)=\left\{\begin{array}{cl}0 & \text { for } T A P_{k}\left(T_{k}, S_{k}\right) \leq L^{k} \\ \frac{\operatorname{TAP}_{k}\left(T_{k}, S_{k}\right)-L^{k}}{U^{k}-L^{k}} & \text { for } L^{k} \leq \operatorname{TAP}_{k}\left(T_{k}, S_{k}\right) \leq U^{k} \\ 1 & \text { for } \operatorname{TAP}_{k}\left(T_{k}, S_{k}\right) \geq U^{k}\end{array}\right.$ 
DOI: 10.14807/ijmp.v11i3.1083

for $k=1,2, \ldots, n$.

4.1. 4.1 Fuzzy non-linear programming problem (FNLP) method based on max-min operator

Fuzzy non-linear programming problem (FNLP) is formulated as follows

$\operatorname{Max} \alpha$

Subject to

$T A P_{k}\left(T_{k}, S_{k}\right)-\alpha\left(U^{k}-L^{k}\right) \leq L^{k}$, for $k=1,2, \ldots, n$.

$0 \leq \alpha \leq 1$

and same constraints and restrictions as the problem (10).

4.2. Fuzzy additive goal programming problem (FAGP) method based on maxadditive operator

Fuzzy additive goal programming problem (FAGP) is formulated as follows

$\operatorname{Max} \sum_{k=1}^{n} \frac{T A P_{k}\left(T_{k}, S_{k}\right)-L^{k}}{U^{k}-L^{k}}$

Subject to

$0 \leq \mu_{T_{A P}}\left(\operatorname{TAP}_{k}\left(T_{k}, S_{k}\right)\right) \leq 1$, for $k=1,2, \ldots, n$.

and same constraints and restrictions as the problem (10).

The non-linear programming problems (12) and (13) can be solved by suitable mathematical programming algorithm.

\section{WEIGHTED FUZZY PROGRAMMING TECHNIQUE (BASED ON MAX-MIN AND MAX-ADDITIVE OPERATORS) TO SOLVE MOIM}

Let us consider positive weights $\omega_{k}$ for each objective $\operatorname{TAP}_{k}\left(T_{k}, S_{k}\right), k=$ $1,2, \ldots, n$ where $\sum_{k=1}^{n} \omega_{k}=1$.

Using the above membership functions (11) and weights $\omega_{k}(k=1,2, \ldots, n)$, we formulate the following programming problems.

5.1. Weighted fuzzy non-linear programming problem (WFNLP) method based on max-min operator

Weighted fuzzy non-linear programming problem (WFNLP) is formulated as follows 
ISSN: 2236-269X

DOI: 10.14807/ijmp.v11i3.1083

$\operatorname{Max} \alpha$

Subject to,

$\omega_{k} \mu_{T A P_{k}}\left(\operatorname{TAP}_{k}\left(T_{k}, S_{k}\right)\right) \geq \alpha$, for $k=1,2, \ldots, n$

$0 \leq \alpha \leq 1$

$\sum_{k=1}^{n} \omega_{k}=1$

and same constraints and restrictions as the problem (10)

\subsection{Weighted Fuzzy additive goal programming problem (WFAGP) method based on max-additive operator}

Weighted fuzzy additive goal programming problem (WFAGP) is formulated as follows

$\operatorname{Max} \sum_{k=1}^{n} \omega_{k} \mu_{T A P_{k}}\left(T A P_{k}\left(T_{k}, S_{k}\right)\right)$

Subject to,

$0 \leq \mu_{T_{A P}}\left(T A P_{k}\left(T_{k}, S_{k}\right)\right) \leq 1$, for $k=1,2, \ldots, n$.

$\sum_{k=1}^{n} \omega_{k}=1$

and same constraints and restrictions as the problem (10)

The non-linear programming problems (14) and (15) can be solved by suitable mathematical programming algorithm.

\section{Numerical Example}

Let us consider an inventory model which consist two items with following parameter values in proper units. Also consider the total storage area is $W=600$ square unit and $\lambda_{1}=$ $0.01, \lambda_{2}=0.02$.

Table 1: Input imprecise data for shape parameters

\begin{tabular}{|c|c|c|c|}
\hline \multicolumn{2}{|l|}{ Item I } & \multicolumn{2}{|l|}{ Item II } \\
\hline Parametric Value in Fuzzy Number & $\begin{array}{l}\text { Defuzzification } \\
\text { of fuzzy number }\end{array}$ & Parametric Value in Fuzzy Number & $\begin{array}{l}\text { Defuzzification } \\
\text { of fuzzy number }\end{array}$ \\
\hline $\begin{array}{l}\widetilde{{c_{1}}}=(400,450,500,550 ; 0.9) \\
\widetilde{a_{1}}=(550,600,650,700 ; 0.96) \\
\widetilde{b_{1}}=(2.5,3,3.5,4 ; 0.92) \\
\widetilde{\alpha_{1}}=(2,3,4,5 ; 0.7) \\
\widetilde{d_{1}}=(38,40,42,44 ; 0.8) \\
\widetilde{\theta_{1}}=(0.01,0.02,0.03,0.04 ; 0.8) \\
\widetilde{P_{1}}=(40,42,44,46 ; 0.9)\end{array}$ & $\begin{array}{l}\widehat{c_{1}}=427.5 \\
\widehat{a_{1}}=600 \\
\widehat{b_{1}}=2.99 \\
\widehat{\alpha_{1}}=2.45 \\
\widehat{d_{1}}=32.8 \\
\widehat{\theta_{1}}=0.02 \\
\widehat{P_{1}}=38.7\end{array}$ & $\begin{aligned} \widetilde{c_{2}} & =(450,500 ; 550,600 ; 0.8) \\
\widetilde{a_{2}} & =(560,610,660,710 ; 0.96) \\
\widetilde{b_{2}} & =(3,3.5,4,4.5 ; 0.93) \\
\widetilde{\alpha_{2}} & =(1,3,4,5 ; 0.8) \\
\widetilde{d_{2}} & =(40,41,42,43 ; 0.8) \\
\widetilde{\theta_{2}} & =(0.01,0.02,0.03,0.04 ; 0.8)) \\
\widetilde{P_{2}} & =(41,42,43,44 ; 0.9)\end{aligned}$ & $\begin{array}{l}\widehat{c_{2}}=420 \\
\widehat{a_{2}}=609.6 \\
\widehat{b_{2}}=3.4875 \\
\widehat{\alpha_{2}}=2.6 \\
\widehat{\theta_{2}}=33.2 \\
\widehat{P_{2}}=38.02\end{array}$ \\
\hline
\end{tabular}


INDEPENDENT JOURNAL OF MANAGEMENT \& PRODUCTION (IJM\&P)

http://www.ijmp.jor.br

v. 11, n. 3, May-June 2020

ISSN: 2236-269X

DOI: 10.14807/ijmp.v11i3.1083

$\widetilde{w_{1}}=(4,5,6,7 ; 0.91)$

$\widehat{w_{1}}=5.005$

$\widetilde{w_{2}}=(3,4,5,6 ; 0.89)$

$\widehat{w_{2}}=4.005$

Table 2: Optimal solutions of MOIM (10)

\begin{tabular}{|c|c|c|}
\hline Methods & $\operatorname{TAP}_{1}\left(T_{1}^{*}, S_{1}^{*}\right)$ & $\operatorname{TAP}_{2}\left(T_{2}^{*}, S_{2}^{*}\right)$ \\
\hline FNLP & 18085.52 & 14482.83 \\
\hline FAGP & 18081.19 & 14487.72 \\
\hline
\end{tabular}

The above Table 2 shows that both FNLP and FAGP methods give almost same results.

The optimal solutions of the MOIM by WFNLP method with different weights are shown in table 3.

Table 3: Optimal solutions of MOIM (10) with different weights by WFNLP method

\begin{tabular}{|c|c|c|}
\hline Weights & $\operatorname{TAP}_{1}\left(T_{1}^{*}, S_{1}^{*}\right)$ & $\operatorname{TAP}_{2}\left(T_{2}^{*}, S_{2}^{*}\right)$ \\
\hline$\left(\omega_{1}=0.5, \omega_{2}=0.5\right)$ & 18085.53 & 14482.84 \\
\hline$\left(\omega_{1}=0.8, \omega_{2}=0.2\right)$ & 17794.61 & 14640.87 \\
\hline$\left(\omega_{1}=0.2, \omega_{2}=0.8\right)$ & 18214.18 & 14149.66 \\
\hline
\end{tabular}

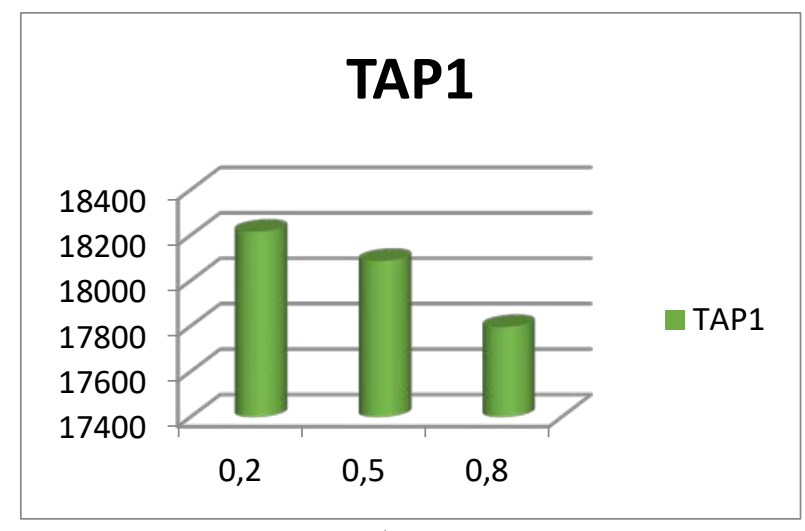

Figure 2: Profit of $1^{\text {st }}$ item with respect to the different weights (WFNLP method)

\section{TAP2}

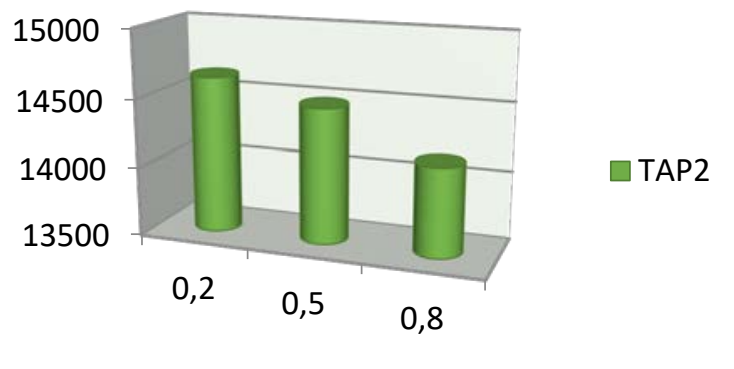

Figure 3: Profit of $2^{\text {nd }}$ item with respect to the different weights (WFNLP method)

Table 4: Optimal solutions of MOIM (10) with different weights by WFAGP method

\begin{tabular}{|c|c|c|}
\hline Weights & $T A P_{1}\left(T_{1}^{*}, S_{1}^{*}\right)$ & $T A P_{2}\left(T_{2}^{*}, S_{2}^{*}\right)$ \\
\hline$\left(\omega_{1}=0.5, \omega_{2}=0.5\right)$ & 18089.58 & 14497.67 \\
\hline$\left(\omega_{1}=0.8, \omega_{2}=0.2\right)$ & 18175.47 & 14205.92 \\
\hline$\left(\omega_{1}=0.2, \omega_{2}=0.8\right)$ & 17846.26 & 14603.66 \\
\hline
\end{tabular}

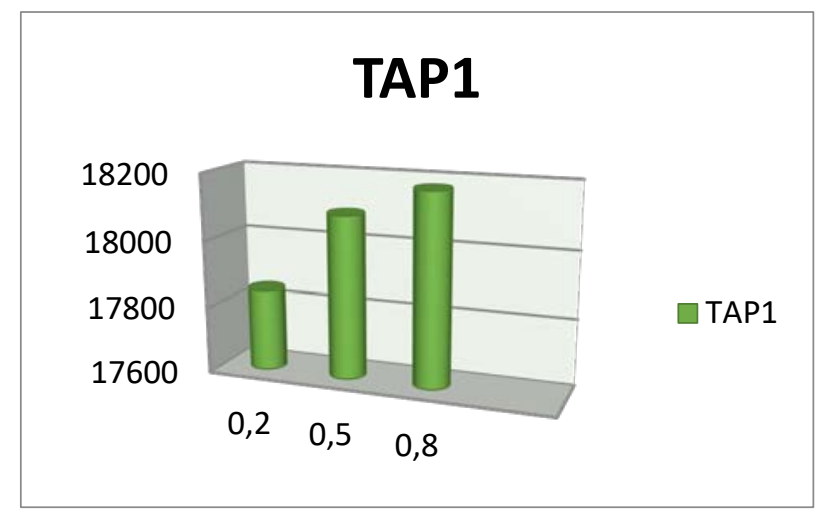

Figure 4: Profit of $1^{\text {st }}$ item with respect to the different weights (WFAGP method)

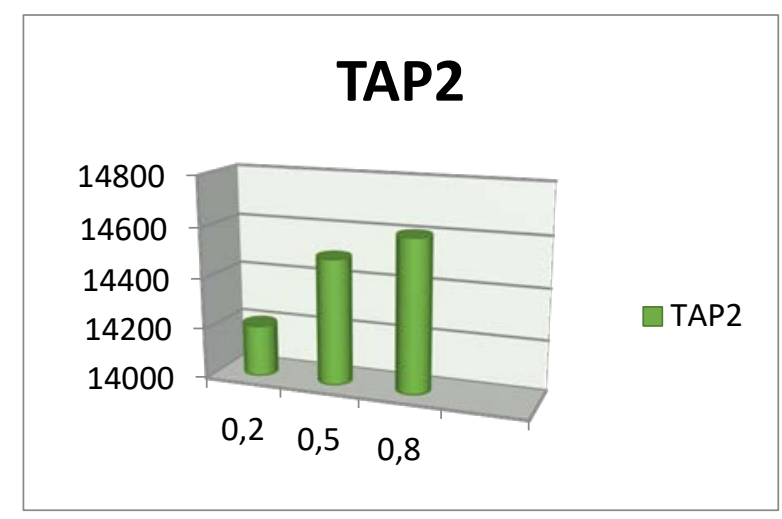

Figure 5: Profit of $2^{\text {nd }}$ item with respect to the different weights (WFAGP method) 


\section{INDEPENDENT JOURNAL OF MANAGEMENT \& PRODUCTION (IJM\&P)}

http://www.ijmp.jor.br

v. 11, n. 3, May-June 2020

ISSN: 2236-269X

DOI: 10.14807/ijmp.v11i3.1083

From the above table 3 and table 4 shows that total profit for $1^{\text {st }}$ and $2^{\text {nd }}$ items in all types are almost same.

\section{SENSITIVITY ANALYSIS}

In the sensitivity analysis the MOIM (10) is solved by using FNLP and FAGP methods for different values of $\theta, a, b, \lambda$ are given in Table 5, 6,7 and 8 respectively.

Table 5: Optimal solutions of MOIM by FNLP \& FAGP methods for different values of $\theta$

\begin{tabular}{|c|c|c|c|}
\hline Methods & $\theta$ & $T A P_{1}\left(T_{1}^{*}, S_{1}^{*}\right)$ & $\operatorname{TAP}_{2}\left(T_{2}^{*}, S_{2}^{*}\right)$ \\
\hline \multirow{4}{*}{ FNLP } & 0.02 & 18085.52 & 14482.83 \\
\cline { 2 - 4 } & 0.04 & 18062.70 & 14466.73 \\
\cline { 2 - 4 } & 0.06 & 18009.48 & 14399.27 \\
\cline { 2 - 4 } & 0.08 & 17955.12 & 14257.89 \\
\hline \multirow{5}{*}{ FAGP } & 0.02 & 18081.19 & 14487.72 \\
\cline { 2 - 4 } & 0.04 & 18060.12 & 14474.77 \\
\cline { 2 - 4 } & 0.06 & 18043.72 & 14448.96 \\
\cline { 2 - 4 } & 0.08 & 18006.95 & 14410.01 \\
\hline
\end{tabular}

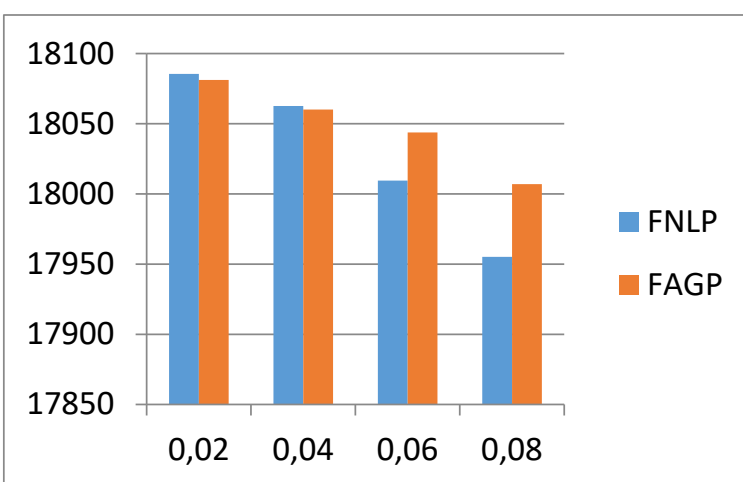

Figure 6: Sensitivity analysis for profit of $1^{\text {st }}$ item w.r.t. $\theta$ by FNLP \& FAGP methods

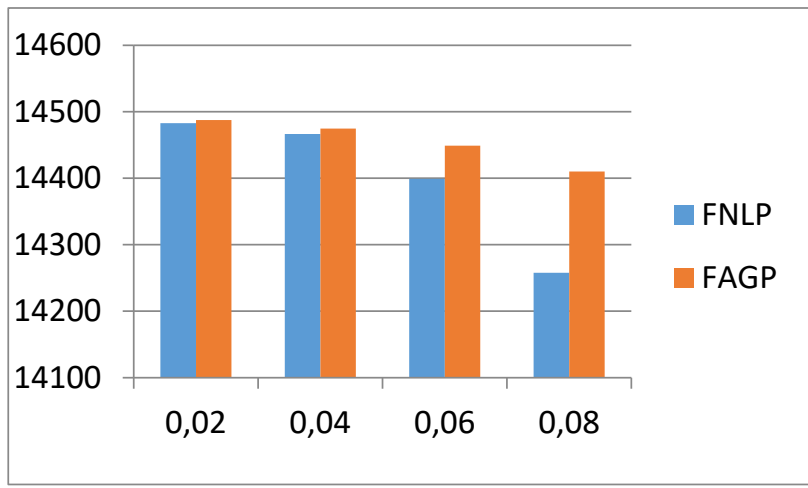

Figure 7: Sensitivity analysis for profit of $2^{\text {nd }}$ item w.r.t. $\theta$ by FNLP \& FAGP methods

From the above figures $6 \& 7$ shows that profit of the both items is decreased when $\theta$ is increased in both methods.

Table 6: Optimal solutions of MOIM (10) by FNLP \& FAGP methods for different values of

\begin{tabular}{|c|c|c|c|}
\hline \multicolumn{4}{|c|}{$a_{1}, a_{2}}$. \\
\hline Methods & $a_{1}, a_{2}$ & $T A P_{1}\left(T_{1}^{*}, S_{1}^{*}\right)$ & $\operatorname{TAP}_{2}\left(T_{2}^{*}, S_{2}^{*}\right)$ \\
\hline \multirow{4}{*}{ FNLP } & $\begin{array}{c}a_{1}=600 \\
a_{2}=609.6\end{array}$ & 18085.52 & 14482.83 \\
\hline & $\begin{array}{c}a_{1}=650 \\
a_{2}=659.6\end{array}$ & 22341.45 & 18069.17 \\
\hline & $\begin{array}{c}a_{1}=700 \\
a_{2}=709.6\end{array}$ & 22987.17 & 21854.66 \\
\hline & $\begin{array}{c}a_{1}=750 \\
a_{2}=759.6\end{array}$ & 23513.51 & 25998.57 \\
\hline \multirow[b]{3}{*}{ FAGP } & $\begin{array}{c}a_{1}=600 \\
a_{2}=609.6\end{array}$ & 18081.19 & 14487.72 \\
\hline & $\begin{array}{c}a_{1}=650 \\
a_{2}=659.6\end{array}$ & 22371.25 & 18129.17 \\
\hline & $\begin{array}{c}a_{1}=700 \\
a_{2}=709.6\end{array}$ & 22967.80 & 21865.45 \\
\hline
\end{tabular}


ISSN: $2236-269 X$

DOI: 10.14807/ijmp.v11i3.1083

\begin{tabular}{|l|c|c|c|}
\hline & $a_{1}=750$, & 23589.23 & 25984.34 \\
$a_{2}=759.6$ & & \\
\hline
\end{tabular}

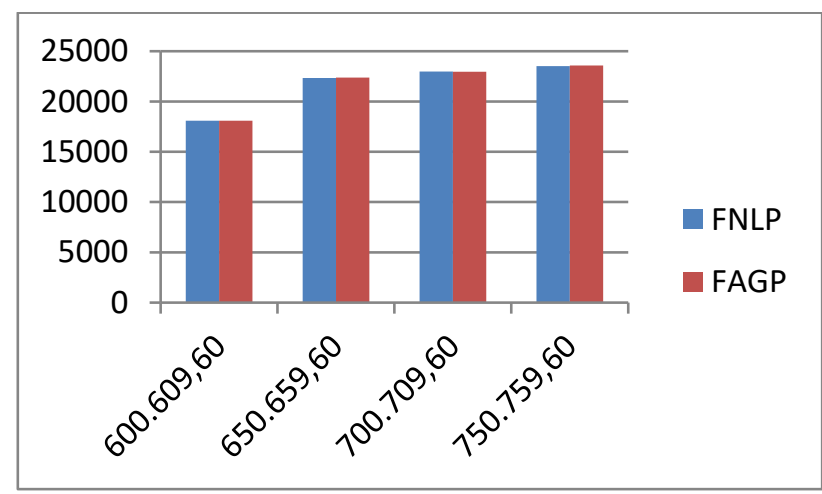

Figure 8: Sensitivity analysis for profit of $1^{\text {st }}$ item w.r.t. $a_{1}, a_{2}$ by FNLP \& FAGP methods

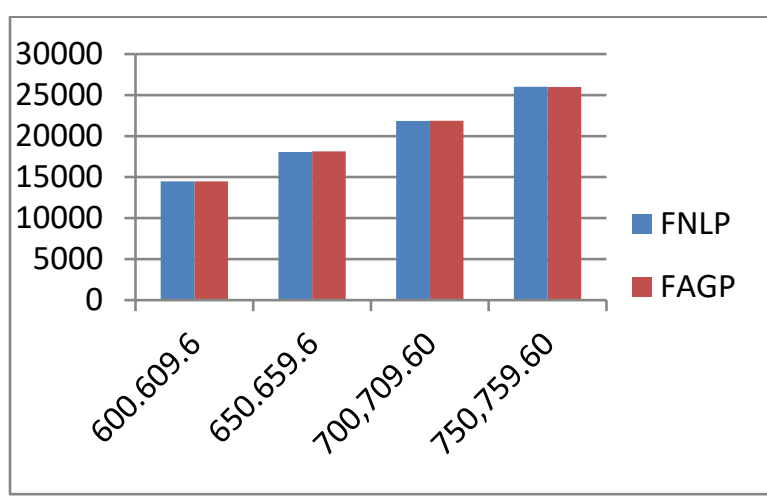

Figure 9: Sensitivity analysis for profit of $2^{\text {nd }}$ item w.r.t. $a_{1}, a_{2}$ by FNLP \& FAGP methods

From the above figures $8 \& 9$ shows that profit of the both items is increased when values of $a_{1}, a_{2}$ are increased in all methods.

Table 7: Optimal solutions of MOIM (10) by FNLP \& FAGP methods for different values of

\begin{tabular}{|c|c|c|c|}
\hline & & $b_{1}, b$ & \\
\hline Methods & $b_{1}, b_{2}$ & $T A P_{1}\left(T_{1}^{*}, S_{1}^{*}\right)$ & $T A P_{2}\left(T_{2}^{*}, S_{2}^{*}\right)$ \\
\hline \multirow{4}{*}{ FNLP } & $\begin{array}{l}b_{1}=2.99 \\
b_{2}=3.49\end{array}$ & 18085.02 & 14482.64 \\
\hline & $\begin{array}{l}b_{1}=3.09 \\
b_{2}=3.59\end{array}$ & 17300.56 & 13934.35 \\
\hline & $\begin{array}{l}b_{1}=3.19 \\
b_{2}=3.69\end{array}$ & 16432.94 & 13285.35 \\
\hline & $\begin{array}{l}b_{1}=3.29 \\
b_{2}=3.79\end{array}$ & 15621.16 & 12673.22 \\
\hline \multirow{4}{*}{ FAGP } & $\begin{array}{l}b_{1}=2.99 \\
b_{2}=3.49\end{array}$ & 18081.15 & 14487.65 \\
\hline & $\begin{array}{l}b_{1}=3.09 \\
b_{2}=3.59\end{array}$ & 17312.43 & 13954.35 \\
\hline & $\begin{array}{l}b_{1}=3.19 \\
b_{2}=3.69\end{array}$ & 16467.87 & 13285.47 \\
\hline & $\begin{array}{l}b_{1}=3.29 \\
b_{2}=3.79\end{array}$ & 15643.45 & 12689.28 \\
\hline
\end{tabular}

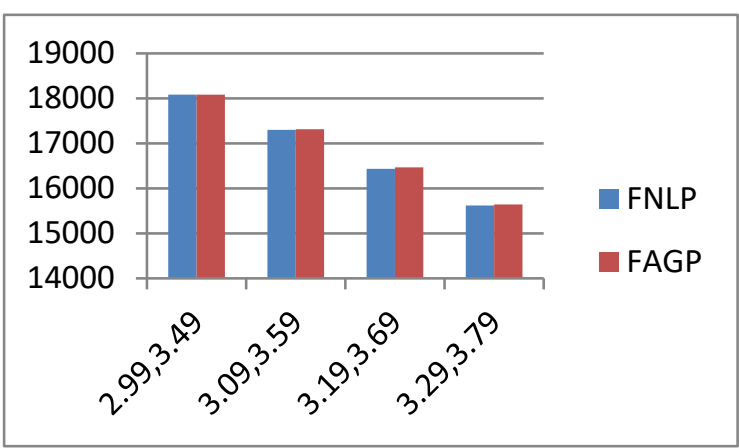

Figure 10: Sensitivity analysis for profit of $1^{\text {st }}$ item w.r.t. $b_{1}, b_{2}$ by FNLP \& FAGP methods

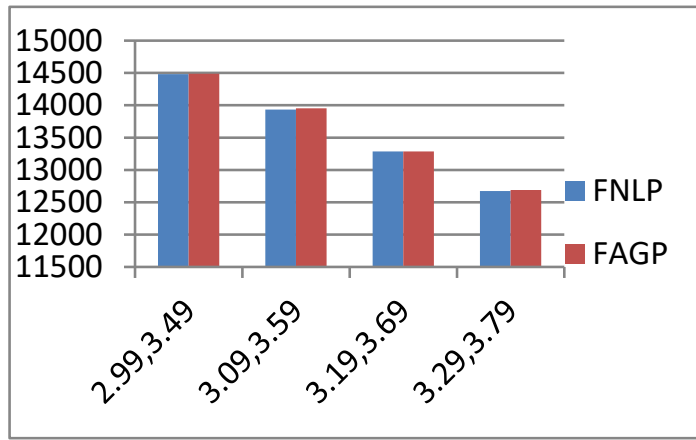

Figure 11: Sensitivity analysis for profit of $2^{\text {nd }}$ item w.r.t. $b_{1}, b_{2}$ by FNLP \& FAGP methods 
INDEPENDENT JOURNAL OF MANAGEMENT \& PRODUCTION (IJM\&P)

http://www.ijmp.jor.br

v. 11, n. 3, May-June 2020

ISSN: 2236-269X

DOI: 10.14807/ijmp.v11i3.1083

From the above figures $10 \& 11$ shows that profit of the both items is decreased when $b_{1}, b_{2}$ are increased in all methods.

Table 8: Optimal solutions of MOIM (10) by FNLP \& FAGP for different values of $\lambda_{1}, \lambda_{2}$

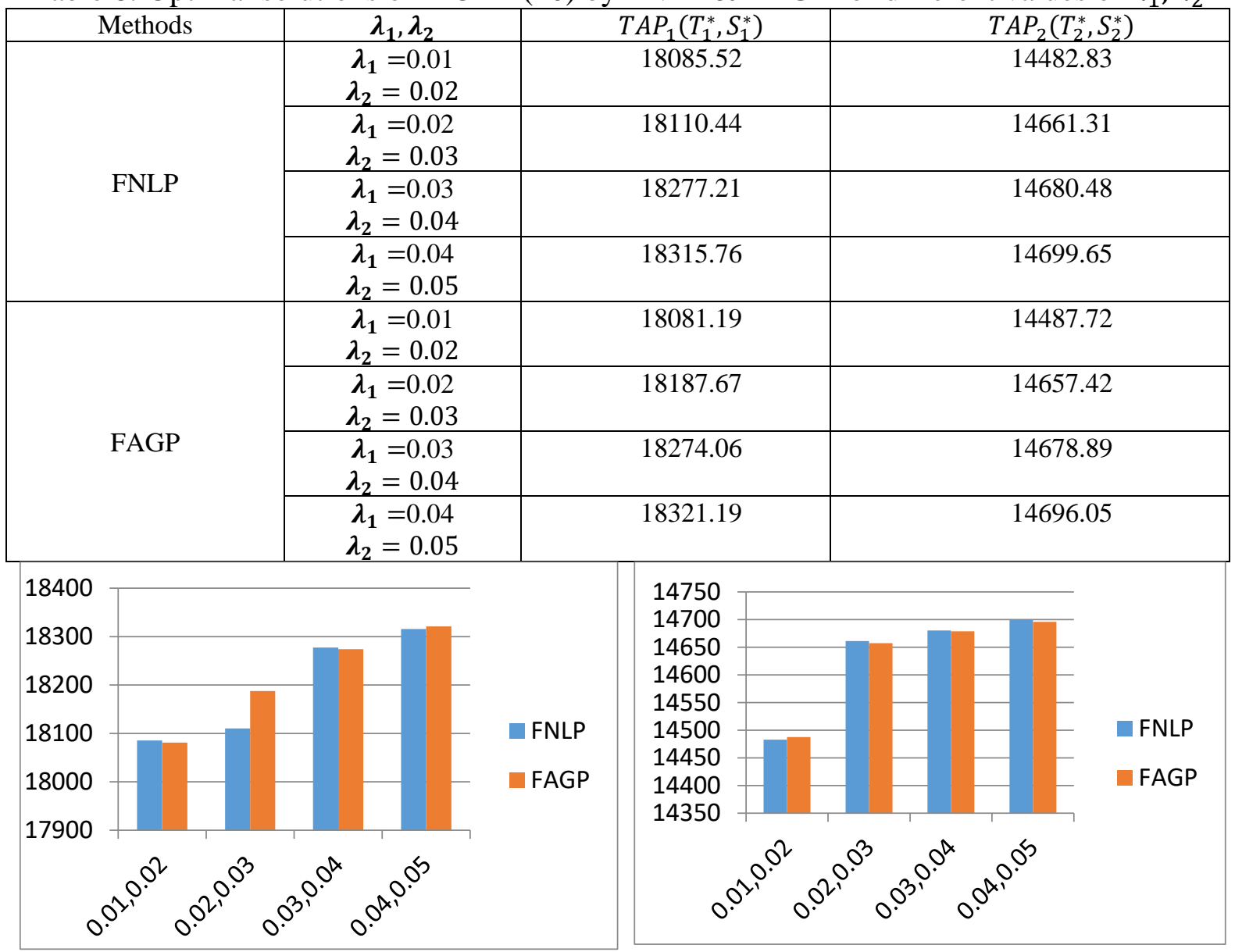

Figure 12: Sensitivity analysis for profit of $1^{\text {st }}$ item w.r.t. $\lambda_{1}, \lambda_{2}$ by FNLP $\&$ FAGP methods

Figure 13: Sensitivity analysis for profit of $2^{\text {nd }}$ item w.r.t. $\lambda_{1}, \lambda_{2}$ by FNLP \& FAGP methods

From the above figures $12 \& 13$ shows that profit of the both items is increased when $\lambda_{1}, \lambda_{2}$ are increased in all methods.

\section{CONCLUSIONS}

In this paper, we have developed a real life inventory model in which time dependent holding cost, selling price as well as time dependent demand. Multi-item inventory has been considered under limitation on storage space. First crisp model is formed then it transferred to the fuzzy model due to uncertainty of cost parameters. All fuzzy cost parameters are taken as generalized trapezoidal fuzzy number. The proposed multi-objective inventory model is solved by using FNLP, FAGP, WFNLP and WFAGP methods.

In the future study, it is hoped to further incorporate the proposed model into more realistic assumption, such as probabilistic demand, introduce shortages etc. Also other type of 
INDEPENDENT JOURNAL OF MANAGEMENT \& PRODUCTION (IJM\&P)

http://www.ijmp.jor.br

v. 11, n. 3, May-June 2020

ISSN: 2236-269X

DOI: 10.14807/ijmp.v11i3.1083

membership functions like as triangular fuzzy number, Parabolic flat Fuzzy Number $(P f F N)$, Parabolic Fuzzy Number ( $p F N$ ) etc. can be used to form the fuzzy model.

\section{ACKNOWLEDGEMENTS:}

The authors are thankful to University of Kalyani for providing financial assistance through DST-PURSE (Phase-II) Programme. The authors are grateful to the reviewers for their comments and suggestions.

\section{REFERENCES:}

ALFARES, H. K.; GHAITHAN, A. M. (2016) Inventory and Pricing Model with PriceDependent Demand, Time-Varying Holding Cost, and Quantity Discounts, Computers \& Industrial Engineering, doi: http://dx.doi.org/10.1016/j.cie.2016.02.009

BHUNIA, A. K.; SHAIKH, A. A. (2014) A deterministic inventory model for deteriorating items with selling price dependent demand and three-parameter Weibull distributed deterioration, International Journal of Industrial Engineering Computations, n. 5, p. 497-510

BORTOLAN, G.; DEGANI R. (1985) A review of some methods for ranking fuzzy numbers, Fuzzy Sets and Systems, n. 15, p. 1-19.

COVERT, R. P.; PHILIP, G. C. (1973) An EOQ model for items with Weibull distribution deterioration. AIIE Transaction, n. 5, p. 323-326.

GHARE, P. M.; SCHRADER, G. H. (1963) A model for exponentially decaying inventory system. International Journal of Production Research, n. 21, p. 449-460.

GHOSH, S. K.; SARKAR , T.; CHAUDHURI, K. (2015) A Multi-Item Inventory Model for Deteriorating Items in Limited Storage Space with Stock-Dependent Demand, American Journal of Mathematical and Management Sciences, v. 34, n. 2, p. 147-161, DOI: 10.1080/01966324.2014.980870.

HARRI, F. W. (1913) How many parts to make at once factory, Mag. Mannage., n. 10, p.135-136.

ISLAMM, S.; ROY, T. K. (2006) A fuzzy EPQ model with flexibility and reliability consideration and demand depended unit production cost under a space constraint: A fuzzy geometric programming approach, Applied Mathematics and Computation, v. 176, n. 2, p. 531-544.

ISLAMM, S.; MANDAL, W. A. (2017) A Fuzzy Inventory Model (EOQ Model) with Unit Production Cost, Time Depended Holding Cost, Without Shortages Under a Space Constrain: A Fuzzy Parametric Geometric Programming (FPGP) Approach, Independent Journal of Management Production, v. 8, n. 2, p. 299-318. DOI: dx.doi.org/10.14807/ijmp.v8i2.535.

ISLAMM, S.; MONDAL, W. A. (2017) Fuzzy E.O.Q Model with Constant Demand and Shortages: A Fuzzy Signomial Geometric Programming (FSGP) Apprach, Independent Journal Of Management \& Production, v. 8, n. 4, p. 1191-1209.

KUMAR, S.; SINGH, A. K.; PATEL, M. K. (2016) Optimization of Weibull deteriorating items inventory model under the effect of price and time dependent demand with partial backlogging, Sadhana, v. 41, DOI 10.1007/s12046-016-0533-4 
LIANG, Y.; ZHOU, F. (2011) A two warehouse inventory model for deteriorating items under conditionally permissible delay in Payment, Appl. Math. Model, n. 35, p. 2221-2231. LIOU T. S.; WANG, M. J. (1992) Ranking fuzzy numbers with integral value, Fuzzy Sets and Systems, n. 50, p. 247-255.

MAITY, M. K. (2008) Fuzzy inventory model with two ware house under possibility measure in fuzzy goal, Euro. J Oper. Res, n. 188, p. 746-774.

MOMDAL, B.; BHUNIA, A. K.; MAITI, M. (2003) An inventory system of ameliorating items for price dependent demand rate. Computers and Industrial Engineering, n. 45, p. 443-456.

ROY, A. (2014) Fuzzy inventory model for deteriorating items with price dependent demand, International Journal of Management Science and Engineering Management, DOI: 10.1080/17509653.2014.959086.

ROY, T. K.; MAITY, M. (1995) A fuzzy inventory model with constraints, Operation research, v. 32, n. 4, p.287- 298.

SHAH, N. H.; SHAH, B. J.; WEE, H. M. (2009) A lot size inventory model for the Weibull distributed deterioration rate with discounted selling price and stock-dependent demand, Int. J. Data Analysis Techniques and Strategies, v. 1, n. 4, p. 355-363.

SRIDEVI, G.; NIRUPAMA DEVI, K.; SRINIVASA RAO, K. (2010) Inventory model for deteriorating items with Weibull rate of replenishment and selling price dependent demand, Int. J. Operational Research, v. 9, n. 3, p. 329-349.

YANG, H. L. (2016) Two Warehouse Partial Backlogging inventory model for deteriorating items under inflation, International Journal of Production Economics, n. 103, p. 362-370.

YU-CHUNG TSAO, GWO-JI-SHEEN (2008) Dynamic pricing promotion and replenishment policies for a deteriorating item under permissible delay in payments, Computer \& operation research, n. 35, p. 3562-3580.

ZADEH, L. A. (1965) Fuzzy sets, Information and Control, n. 8, p. 338-353.

ZIMMERMANN, H. J. (1985) Application of fuzzy set theory to mathematical programming, Information Science, n. 36, p. 29-58.

ZIMMERMANN, H. J. (1992) Methods and applications of Fuzzy Mathematical programming, in An introduction to Fuzzy Logic Application in Intelligent Systems, p. 97120, Kluwer publishers, Boston. 